\title{
Éditorial
}

Le volume 20 de la revue Organisations et Territoires se termine en 2011 par la publication du deuxième et du troisième numéro sous la même édition. Ce numéro double propose une série d'articles portant sur des thématiques variées touchant la dimension psychologique de travail, le management de projets, l'entreprenariat, le développement territorial, les modèles de gouvernance et des responsabilités en matière de développement durable.

Jacinthe Douesnard examine les impacts psychologiques de la dangerosité et des facteurs de risque inhérents au métier de pompier. À cette fin, elle propose un modèle mettant en relief certains indicateurs de problèmes de santé tels que la détresse psychologique, l'épuisement professionnel et le stress post-traumatique. Les résultats empiriques de l'étude portent à croire que la dangerosité et les contraintes liées au travail des pompiers n'ont pas d'incidence significative sur l'état de santé psychologique de la grande majorité des pompiers. L'auteure note cependant qu'il est important de déployer les efforts reçus pour remédier aux problèmes vécus par une faible proportion de pompiers ayant des symptômes de trouble de stress posttraumatique, d'épuisement professionnel ou de détresse psychologique.

Imen Latrous propose dans le deuxième article un modèle théorique en vue d'analyser l'effet de la structure de propriété sur le niveau d'endettement de l'entreprise. Grâce aux données obtenues à partir d'un échantillon d'entreprises cotées à la bourse de Paris, l'étude démontre que lorsque les actionnaires de contrôle détiennent des faibles parts au capital, ils cherchent alors à accroître leur pouvoir à travers un recours massif à la dette. Autrement dit, les actionnaires de contrôle ont une influence considérable sur la politique de financement de l'entreprise.

Le troisième article proposé par Maripier Tremblay, Josée Audet et Yvon Gasse dresse le portrait des aspirants-entrepreneurs de la génération Y. Après avoir décrits les caractéristiques des personnes pouvant faire partie de la génération $\mathrm{Y}$ en tant que citoyens, employés et entrepreneurs, les auteur(e)s ont réalisé une analyse de contenu de 59 demandes de bourses présentées par des étudiants du profil entrepreneurial de 1'Université Laval. La synthèse des données, colligées pour les fins de cette étude, démontre que les membres de la génération Y aspirent à créer une entreprise pour des besoins de réalisation de soi et d'autonomie. La génération Y semble se démarquer des générations antérieures dans le domaine de l'entrepreneuriat. Les résultats préliminaires montrent que le portrait dégagé est cohérent avec les caractéristiques généralement attribuées aux Y dans la littérature : familiarité avec les voyages; implication sociale, communautaire et environnementale; besoin d'accomplissement et recherche d'autonomie; prépondérance de projets collectifs ou en équipe présentant une dimension sociale et environnementale. Ces constats amènent à réfléchir aux changements à apporter pour adapter les méthodes d'enseignement et les formes traditionnelles d'accompagnement aux besoins particuliers de ce nouveau type d'entrepreneur.

Le quatrième article présente une étude de la place des femmes entrepreneures dans l'ensemble de l'économie et plus particulièrement dans la nouvelle économie du savoir. Les auteurs(e)s Sylvie Paré et Kelogue Therasme ont en effet réalisé une analyse comparative de la situation des femmes entrepreneures appartenant à des groupes d'origine immigrante et occupant des fonctions de responsabilité entrepreneuriale. Les résultats obtenus grâce à la synthèse de données extraites des Répertoires Scott démontrent une très faible présence des femmes à la tête d'entreprises dans le domaine de l'économie du savoir. Cependant, l'étude montre que les femmes provenant des groupes des minorités ethniques sont davantage présentes en tant qu'entrepreneure au sein de la nouvelle économie axée sur le savoir.

Geoffrey Kiel, James Beck et Jacques Grisé proposent dans le cinquième article une approche 
originale de l'évaluation des conseils d'administration. Dans le but d'améliorer les pratiques de gouvernance et de l'efficacité des instances décisionnelles, les auteurs suggèrent un modèle d'évaluation du conseil d'administration comportant sept étapes. Le modèle s'avère un guide pratique en mettant en relief les questions clés que les conseils d'administration doivent prendre en considération lorsqu'ils planifient l'évaluation d'un conseil et des administrateurs. L'analyse réalisée dans le cadre de cet article porte à croire que les conseils d'administration hautement performants ont tendance à adopter un processus continu d'évaluation pour assurer une amélioration de leur rendement.

Le sixième article met en relief les incidences de la sphère juridique sur les modes de gouvernance des organisations et de la gestion des rapports de voisinage dans un contexte de développement durable. En effet, les auteur(e)s Jeanne Simard et Marc-André Morency présentent les implications éthiques de la « responsabilité sans faute », un nouveau régime issu du jugement de la Cour suprême du Canada dans l'affaire Ciment du Saint-Laurent. Grâce à une analyse intéressante du nouveau régime juridique en matière de trouble de voisinage, les auteur(e)s croient qu'une gouvernance participative impliquant toutes les parties prenantes ainsi que les organisations privées s'impose dans un contexte de développement durable où les dimensions économiques, sociales et environnementales doivent être prises en considération simultanément.

Dans le septième article, Martin Simard, Suzanne Tremblay et Carl Brisson analysent l'évolution de la trajectoire de développement de Kénogami, une ville fondée en 1912 par la Compagnie Les Prices Brothers autour d'une papeterie. Les auteur(e)s dressent ainsi le portrait historique, urbanistique et socio-économique. L'étude démontre que le secteur de Kénogami a évolué en se transformant d'une ville modèle à un quartier défavorisé. Compte tenu de ce constat les auteur(e)s ont ensuite présenté les stratégies de revitalisation axées sur la mise en valeur du patrimoine, sur le développement commercial et sur le développement communautaire.
Le huitième article propose une réflexion sur l'articulation entre la gestion des projets et le management stratégique par projet. Les auteures Selma Katlane Ben Mlouka et Hajer Khedher Ouinniche mettent en relief l'importance d'intégrer finement le projet en tant que mode d'organisation du travail, de la production et de l'entreprise très favorable à l'atteinte des orientations stratégiques. Sur ce point, l'article propose un modèle comportant cinq phases pour opérationnaliser la mise en place de la stratégie de l'entreprise à travers les projets dans une optique orientée vers la satisfaction des clients.

Le neuvième article examine l'impact du commerce international sur la réduction de la pauvreté et des inégalités. Thierno Diallo et Patrice Beaulieu proposent un modèle théorique pour mieux comprendre les relations entre ces phénomènes d'une grande actualité. La synthèse de nombreuses données provenant de plusieurs sources a permis de tester les hypothèses à l'étude dans cinq pays, soit l'Inde, le Brésil, le Burkina Faso, le Maroc et la Corée du Sud. Les résultats portent à croire que l'impact du commerce international sur la réduction de la pauvreté et des inégalités est très important dans les trois nouveaux pays industrialisés (Inde, Brésil et Maroc). Cependant, les auteurs notent que cet impact n'est pas systématique. En effet, certaines conditions doivent être présentes dans le cas de pays en développement, tel que le Burkina Faso.

Dans le dixième article, Donatien Avelé et Gérard Fillion explorent les effets des pratiques de contrôle de gestion sur la performance municipale. À cette fin, les auteurs ont proposé un cadre conceptuel en vue de mieux comprendre les déterminants de la performance municipale. Le modèle a été ensuite confronté à la réalité des municipalités camerounaises. Selon les résultats de l'enquête et des tests statistiques, il existe une relation positive entre le degré de décentralisation de la structure municipale et la performance des services publics municipaux. L'étude démontre aussi que les contrôles de gestion sont pour la plupart exercés par les ministères de tutelle. 
Le dernier article, proposé par Carlos LargachaMartinez, s'inscrit dans une démarche de questionnement des aspects éthiques et déontologiques de la fonction managériale. L'auteur suggère un cadre conceptuel mettant en relief les contours d'un leadership humaniste propice à des milieux de travail responsables et socialement acceptables. En effet le "Quantum Humanistic Management » est conçu comme un management alternatif qui accorde une place importante à la dimension humaine des organisations : l'employé, le gestionnaire ou tout autre acteur doivent être au centre des finalités organisationnelles et non comme moyens au service de la production et de la recherche du rendement maximum. Cet article écrit dans la langue de Shakespeare, une première pour la revue Organisations \& Territoires, s'avère très original en ces temps de remise en question des modèles et des pratiques de gestion.

La chronique du livre comporte trois recensions de livres examinant des problématiques variées. Le premier livre met en relief l'importance de la subjectivité en tant que dimension qui guide l'action et les activités quotidiennes de gestion. Le deuxième livre, sous forme d'un roman, traite les diverses facettes, techniques et modalité du coaching. Le troisième ouvrage explore la problématique des aptitudes managériales à piloter le changement organisationnel en temps de crise.

Enfin, ce double numéro se termine par la première partie d'une interview avec Henry Mintzberg, lequel a été invité à présenter sa vision concernant la théorisation et la pratique du management. En fait, il s'agit d'une réactualisation de l'entretien réalisé en 1991 dont les propos demeurent valables et d'une grande actualité. D'abord, le professeur Mintzberg a réaffirmé les idées qu'il a déjà soutenues il y a déjà plus de deux décennies. Il a aussi proposé des réponses originales à certaines nouvelles questions posées lors d'une interview réalisées en novembre 2011. La deuxième partie de l'interview sera publiée dans le prochain numéro de la revue Organisations \& Territoires.

Brahim Meddeb, Ph. D. Le Directeur 


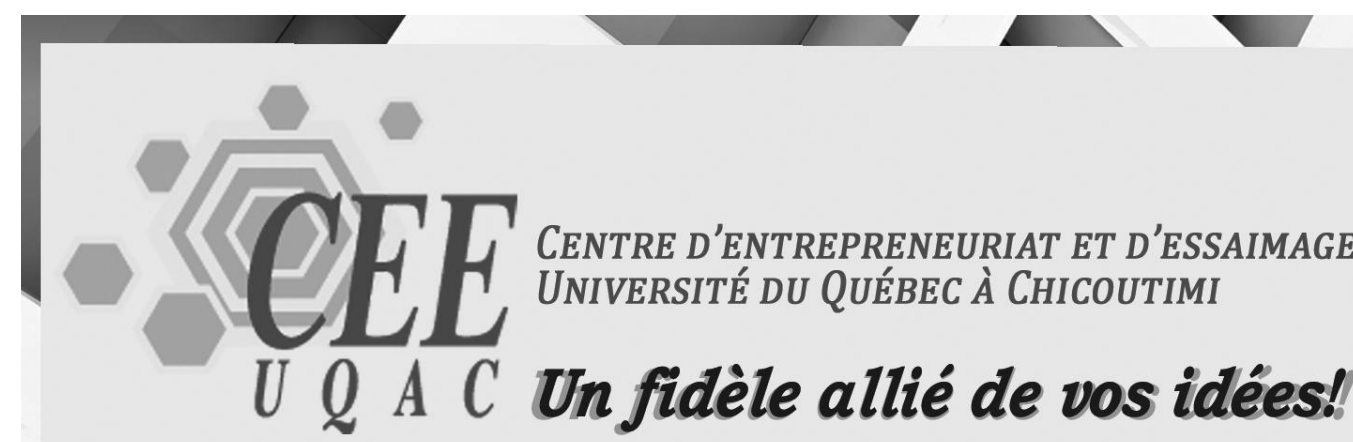

Le CEE-UQAC vous offre :

- Un accompagnement personnalisé en création, démarrage et relève d'entreprise!

- Validation d'idées d'affaires

- Soutien à la réalisation du plan d'affaires

- Des activités à saveur entrepreneuriale!

- Midi-conférences, colloques, salons

- Ateliers d'information et de perfectionnement

- Chroniques entrepreneuriales

- Concours entrepreneuriaux

- Des projets technologiques rémunérés, directement liés à votre champ d'études, qui vous offrent la possibilité de travailler dans les PME régionales!

- Un Réseau international d'affaires à l'UQAC (RIA-UQAC) qui permet aux PME de développer des projets d'affaires avec l'étranger grâce à la collaboration des étudiants internationaux diplômés de l'UQAC!

- Un Centre de développement technologique en jeux vidéo et en informatique (CDT-UQAC) qui contribue à l'émergence de projets innovateurs et au développement d'entreprises en lien avec la conception de jeux vidéo et l'informatique, et ce, par un encadrement d'affaires adapté.

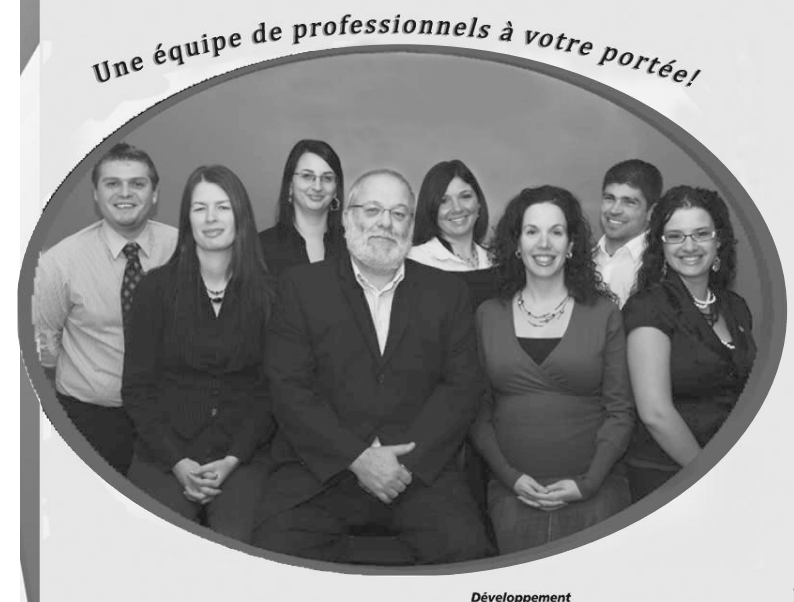

Venez nous rencontrer!

\section{CEE-UQAC Chicoutimi}

555 , boul. de l'Université, local H1-1180

Pavillon des humanités, UQAC

Chicoutimi (Québec) G7H 2B1

Téléphone : $418545-5011$ poste 4655

Courriel : cee-uqa@@uqac.ca

CEE-UQAC Côte-Nord

Cégep de Sept-îles, 175, de la Vérendrye

Sept-Iles (Québec) G4R 5B7

Téléphone : 418 968-8387 • cee_cn@uqac.ca

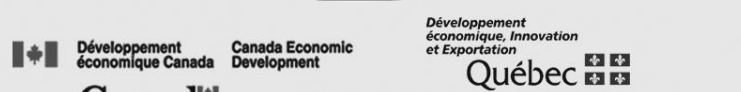

\section{UQAC}

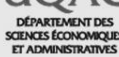

Canadă

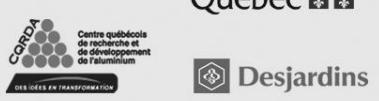

FONDATION

UQAC
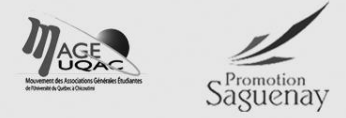